$62^{\text {ème }}$ Congrès de la SFCO, 03004 (2014)

DOI: $10.1051 /$ sfco/20146203004

(C) Owned by the authors, published by EDP Sciences, 2014

\title{
Lichen plan buccal et transformation maligne : dix ans de suivi au Service de Pathologie et Chirurgie Buccales CHU Oran.
}

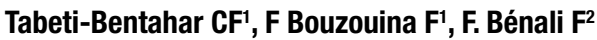 \\ 1. Service de Pathologie et de Chirurgie Buccales, CHU Oran, ALGÉRIE
}

2. Anatomopathologiste libérale, Oran, ALGÉRIE

Le lichen plan (LP) est une dermatose cutanéo-muqueuse inflammatoire chronique, dont la description clinique initiale a été faite par Wilson en 1869 et la description histologique par Dubreuil en 1906 [Lysitsa et al. 2007]. La moitié des sujets ayant des lésions cutanées développent également des lésions buccales, mais $25 \%$ des sujets présentent uniquement des lésions buccales [Setterfield et al. 2000]. Contrairement aux lésions cutanées qui guérissent le plus souvent spontanément en 1 à 2 ans, les lésions muqueuses ont une évolution prolongée sur plusieurs années [Grosshans 2003]. La transformation maligne du lichen plan buccal (LPB) reste un sujet de controverse malgré les nombreuses études qui lui ont été consacrées. Le taux de transformation maligne varie de façon importante selon les études de 0 à $5,3 \%$ [lsmail et al. 2007].

De février 2003 à novembre 2013, les malades présentant un LPB ont été colligés au Service de Pathologie et Chirurgie Buccales CHU Oran avec confirmation clinique et histo-pathologique. Au total, 56 malades ont été répertoriés avec 12 hommes et 44 femmes. L'âge au moment du diagnostic varie de 23 ans à 81 ans. Quarante-deux malades présentaient un LPB isolé dont 09 hommes et 33 femmes. Douze patients présentaient un LPB associé à un LP cutané : 03 hommes et 09 femmes. Une malade présentait un lichen muqueux (buccal et vaginal). Une patiente présentait un lichen cutanéo-muqueux (cutané, buccal et vaginal). Vingt-huit patients présentaient une co-morbidité dont 4 hommes et 24 femmes. Huit malades de l'ensemble des 56 ont développé une tumeur maligne sur lésion de LPB, répartis en trois hommes et cinq femmes. Le décès est survenu chez cinq patients sur les 56 : trois d'entre eux suite aux complications de leur cancer buccal et un suite à une insuffisance respiratoire par tuberculose miliaire pulmonaire avec insuffisance rénale aigüe. La cinquième malade est décédée suite aux complications de son hépatite $C$.

Plusieurs études sur la transformation maligne du LPB ont été publiées à travers le monde. Bornsteïn et al. de 1995 à 2001 en Suisse, sur 145 patients suivis, 4 ont développé une transformation maligne de LPB. Dans l'étude de Mignogna et al., en 2007 en Italie, sur 45 patients présentant un LPB ayant développé une dysplasie sévère ou un carcinome épidermoïde, 3 patients sont décédés suite à leur atteinte buccale. Van der Meij et al. en 2007 aux Pays Bas ; sur 192 malades, suivis entre 7,6 et 96,9 mois ; quatre d'entre eux ont développé un carcinome épidermoïde de la muqueuse buccale durant leur suivi. Dans l'étude de Fang et al., en Chine entre 1951 à 2006, sur 2.119 malades présentant un LPB, 23 ont développé un carcinome épidermoïde. Bombeccari et al., en 2011 dans une étude de cohorte en Italie, rapportent une cancérisation chez 8 malades sur les 327 suivis pendant environ 7 ans. Dans la présente série, sur 56 malades et en dix ans, 8 transformations malignes ont été notées.

Les observations présentées prouvent que le LPB est un réel désordre potentiellement malin dont la surveillance clinique doit être régulière.

TABETI-BENTAHAR Cherifa taberi_cherifa@yahoo.fr

This is an Open Access article distributed under the terms of the Creative Commons Attribution License 4.0, which permits unrestricted use, distribution, and reproduction in any medium, provided the original work is properly cited. 